\title{
Beamline bonanza for Japanese researchers
}

\section{A proton-accelerator complex will incorporate one of the world's most intense pulsed neutron sources.}

\section{TOKAIMURA, JAPAN}

Staff at the new Japan Proton Accelerator Research Complex (J-PARC), in the seaside village of Tokaimura in Ibaraki, like to use familiar analogies to introduce the facility. The 330-metre-long building that houses the linear proton accelerator - the engine behind the complex - is similar in size to Tokyo's train station. And the main 50-gigaelectronvolt synchrotron, with its three flattened sides and a diameter of 500 metres, resembles an onigiri - the rice ball found in every convenience store throughout Japan.

J-PARC will support several fields in which Japan has excelled, and if organizers have their way, Japan will stay at the forefront of them. Starting next month, the facility will accelerate and smash protons into a variety of targets to produce beams of subatomic particles such as neutrons, muons, neutrinos and kaons. It promises to provide its users - including a large proportion of international scientists - with new ways to image molecules, probe neutrino physics and more.

Most accelerators are built either to study nuclear physics or fundamental particle physics, or for materials or medical use. J-PARC is designed to do all of that, says director Shoji Nagamiya, in part because it sprang from two earlier proposals that merged. The Tsukubabased High Energy Accelerator Research Organization was looking to build a 50-gigaelectronvolt ring for neutrino and kaon projects; the Japan Atomic Research Energy Institute, which was later reorganized as the Japan Atomic Energy Agency, wanted a neutron beam. The idea to share an accelerator, which came about in 1998, made sense, but the union was consumed by red tape. The institutes finally signed off on what would become a ¥152billion (US\$1.6-billion) collaboration in 2001. "J-PARC is like an indispensable, high-quality single crystal finally grown after a tremendous struggle," says Kazuyoshi Yamada, a materials researcher at Tohoku University in Sendai who started preparing samples for it two years ago.

At the complex's materials and life-science facility, research kicks off in earnest next month when seven neutron beamlines start running. These will be used to precisely image biological molecules and inorganic crystals, among other things. For instance, Nobuo Niimura of Ibaraki University will lead a protein-structure project; researchers from Hitachi will analyse lithium-based materials used for plug-in electric cars (see page 436); and yet others will study the behaviour of magnetic fields inside superconductors.

\section{Neutron sources around the world}

\section{Japan's Proton Accelerator}

Research Complex is not the only powerful neutron source to fire up these days.

In Tennessee, the Spallation Neutron Source at the Oak Ridge National Laboratory is already the world's most intense pulsed neutron source at 630 kilowatts, with a projected ramp-up to 1.4 megawatts in the next two years and a projected top-out at 4 megawatts. Two science instruments are coming online by the end of the year, bringing the total to nine operating instruments.
Not to be left behind, the United Kingdom is refurbishing and adding to its 25-year-old ISIS neutron source. This 160-kilowatt facility already hosts 29 operating instruments, and in August it added a second, £146-million (US\$218-million) proton target station with seven new instruments. In France, the continuous, 58-megawatt neutron source at the Institut Laue-Langevin now has $€ 160$ million (US\$202 million) to upgrade the facility's 40-plus instruments.
As for the future, China could stake its claim with the projected 2015 opening of the 100-kilowatt China Spallation Neutron Source. The Dongguan-based facility is to be ISIS-like in design, with an estimated 1.4-billionrenminbi (US\$205-million) price tag.

But leading the field could be Europe's proposed 5-megawatt European Spallation Source. If project leaders can pick a site for it in the next two months, the $€ 1$.5-billion neutron source could come online in 2022. Ashley Yeager

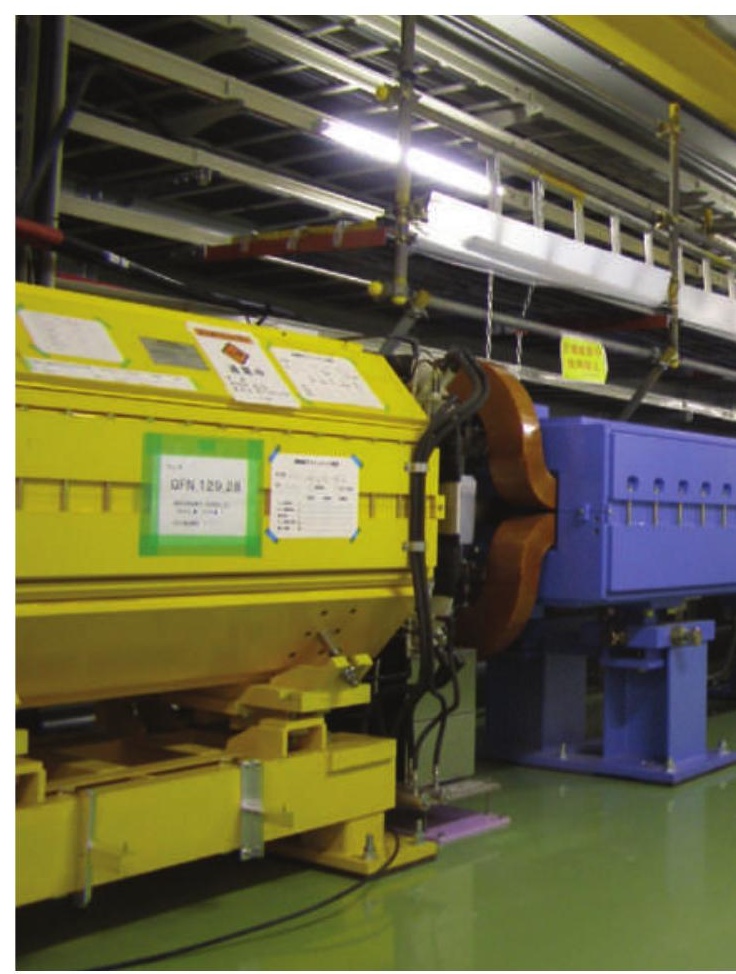

J-PARC's neutron beams will be 1 megawatt - intense enough to dramatically speed up the rate of discovery. Earlier neutron studies, using neutrons produced in nuclear reactors, led to only five or so protein structures being completed in a year, says Niimura. At the new complex, this number will jump to 100 . Thomas Mason, director of the Spallation Neutron Source at the Oak Ridge National Laboratory in Tennessee (see 'Neutron sources around the world'), says that the two facilities are "a new threshold in performance”. In 2009, J-PARC will also start running experiments with the world's most intense pulsed muon beam, exceeding by 30-fold the Rutherford Appleton Laboratory's facility in Didcot, UK.

Meanwhile, the largest and costliest part of the facility - the 50-gigaelectronvolt synchrotron - will be devoted to studies of neutrinos and kaons. A team of some 400 researchers will race to observe for the first time the transition of one variety of neutrinos, called muon neutrinos, into another called electron neutrinos. The observation could offer clues about the range of neutrino masses and about CP violation, a concept key to understanding why matter dominates over antimatter in the Universe.

Groups in China and France are pursuing a similar goal, but with less-energetic neutrinos generated by nuclear reactors. The Fermi National Accelerator Laboratory in Batavia, Illinois, will have a more powerful neutrino source when its NOvA gets up and running after 2014. 


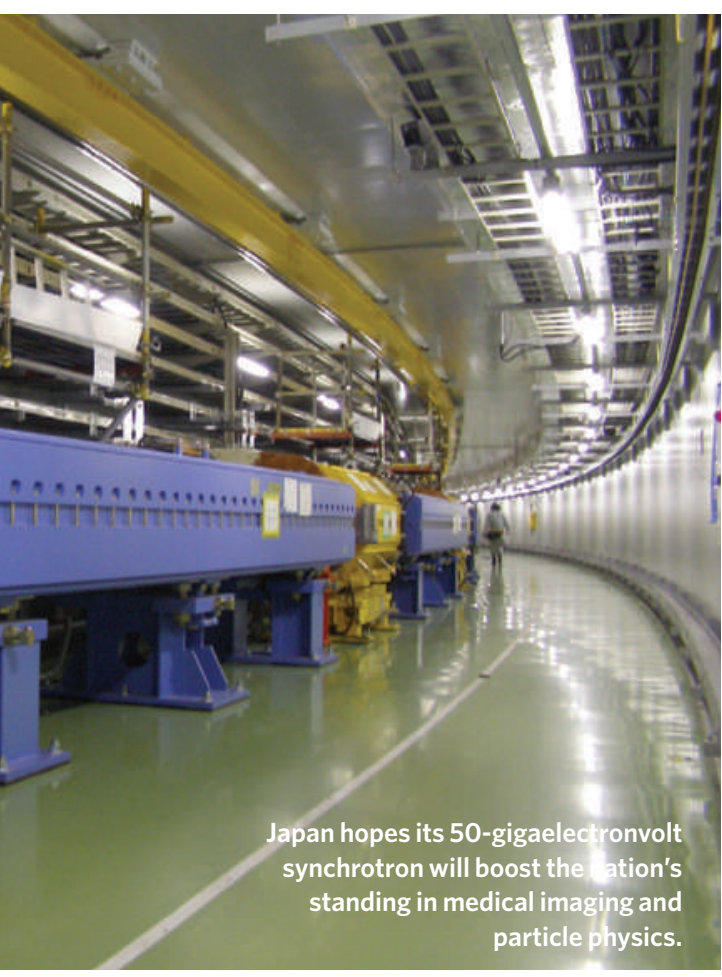

To create the beam of muon neutrinos, J-PARC will send a proton beam at a graphite target. Unlike conventional head-on collisions, J-PARC's proton beam will be adjustable to allow collisions at an angle. The angled collision will lower the average energy, but more of the resulting neutrinos will be in the range likely to oscillate into electron neutrinos on their underground trip to the SuperKamiokande detector, 295 kilometres away.

When J-PARC's big synchrotron isn't doing neutrino physics, it will focus on kaons and hypernuclear experiments. For example, scientists will look for evidence that neutral kaons decay into neutral pions plus a neutrino and an antineutrino.

About half of the $\$ 10$ million needed for the decay experiment, and $\$ 8$ million needed for other kaon experiments, still await funding. But this year's Nobel prizes may help to give the field a boost. In 2002, the awarding of the Nobel to Masatoshi Koshiba, then at the University of Tokyo, for neutrino work meant that Japan ploughed another $¥ 16$ billion into J-PARC’s neutrino project, accelerating its opening. This year, the Nobel physics prize was shared by the High Energy Accelerator Research Organization's Makoto Kobayashi and Kyoto University's Toshihide Maskawa for their work in explaining the dominance of matter over antimatter in the Universe - and some think that might boost funding for the kaon experiments at J-PARC. "It was," says Nagamiya, "very good timing." David Cyranoski

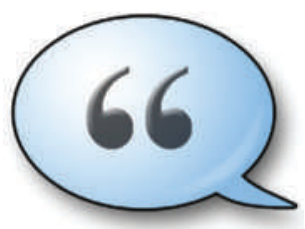

HAVE YOUR SAY

Comment on any of our news stories, online.

www.nature.com/news
European scientists and funding agencies have launched an ambitious plan to prioritize the astronomy projects they believe should be supported over the next 20 years.

The ASTRONET Infrastructure Roadmap was released on 25 November, timed to coincide with the opening of the European Space Agency's (ESA's) ministerial meeting in The Hague, the Netherlands, where, as Nature went to press, ESA member states were due to thrash out how planned space projects will be funded over the coming years. Several large ESA projects - including a suite of Earthobserving satellites called Kopernikus and the proposed ExoMars rover - are already facing funding difficulties (see Nature 455, 840-841 \& 1013; 2008).

ASTRONET was set up in 2005 to deliver a concerted vision from the European astronomy community. It echoes a process that has been undertaken five times in the United States - the National Research Council's 'decadal survey' of astronomy and astrophysics.

Speaking with a single voice has helped US space scientists to get big projects such as the Hubble and Spitzer telescopes off the ground. Astronomer Joseph Taylor at Princeton University, who co-chaired the last decadal survey in 2001, thinks Europe will benefit from having a consolidated approach. "Over the past 50 years it's been a very useful way of prioritizing our national efforts," he says.

The European version collates views from 28 countries, as well as ESA and the European Southern Observatory (ESO). The process is a first for European astronomy, but could happen every five to ten years, says Michael Bode at Liverpool John Moores University, UK, and leader of ASTRONET.

ASTRONET's top priority is the European Extremely Large Telescope, a 42-metre telescope operating in the visible and infrared wavelengths that would be the biggest yet built. The roadmap says that construction should begin at the ESO in 2010, backed by $€ 1$ billion (US\$1.3 billion) in funding that has yet to be committed. The Square Kilometre Array, a radio telescope aiming to probe the early Universe, should be the next priority, and would involve a global consortium of partners.

ESA has its own 'Cosmic Vision' for space-based missions for 2015-25, and there is inevitable overlap with the ASTRONET plan, says Bode. ESA missions favoured by the roadmap include the gravitational-wave observatory LISA - a joint project with NASA - and a probe called Gaia that will map the position and velocity of a billion stars in our Galaxy.

But the roadmap might be bad news for some facilities, because supporting big projects will mean savings must be made elsewhere. ASTRONET is already reviewing the roles of some smaller, twoto four-metre European telescopes in an attempt to eliminate research overlap, for example. "We think it is important to rationalize the observational set-up in Europe," says Jean-Marie Hameury, ASTRONET coordinator and deputy director of the National Institute of

Space Science at the CNRS, France's basic-research agency in Paris. A similar review of eight-metre telescopes should follow, he adds.

One such telescope, the 2.6-metre Nordic Optical Telescope on La Palma in the Canary Islands, is run by ASTRONET board chair Johannes Andersen. He points out that the roadmap's recommendation to integrate space- and
- something not currently done at a European level should produce more science overall.

ASTRONET's plan requires European nations to collectively spend $20 \%$ more than the $€ 2$ billion they spend annually on astronomy. Securing that funding will be "the toughest part of the job", admits Hameury.

Katharine Sanderson 